\title{
Non-Uremic Calciphylaxis: A Rare Diagnosis with Limited Therapeutic Strategies
}

\author{
Felisbela Gomes, Pedro La Feria, Catarina Costa, Rita Santos \\ Unidade Funcional de Medicina 2.1, Hospital de Santo António dos Capuchos, Centro Hospitalar de Lisboa Central, Lisbon, Portugal
}

Received: 03/11/2018

Accepted: 09/11/2018

Published: 04/12/2018

How to cite this article: Gomes F, La Feria P, Costa C, Santos R. Non-uremic calciphylaxis: a rare diagnosis with limited therapeutic strategies. EJCRIM 2018;5: doi:10.12890/2018_000986.

Conflicts of Interests: The Authors declare that there are no competing interests.

This article is licensed under a Commons Attribution Non-Commercial 4.0 License

\section{ABSTRACT}

Calciphylaxis is a rare condition characterized by the emergence of non-healing skin ulcers secondary to arterial calcification and thrombosis, typically diagnosed in patients with end-stage kidney disease (ESKD). When it develops in patients without ESKD, it is called non-uremic calciphylaxis (NUC). The latter is an even rarer diagnosis with an uncertain pathophysiology and a high mortality rate (52\%), mainly due to sepsis (50\%). Cutaneous biopsy is diagnostic. Therapeutic measures recommended for NUC are limited to wound debridement, analgesia, and control of infection and risk factors. Other therapeutic options exist but with a low level of evidence. We present the case of a 78-yearold woman with NUC in her lower limbs who died of sepsis. NUC is a therapeutic challenge lacking efficient strategies.

\section{LEARNING POINTS}

- Calciphylaxis in the absence of end-stage kidney disease is called non-uremic calciphylaxis (NUC).

- This disease is a diagnostic and therapeutic challenge.

- As therapeutic strategies for NUC mainly derive from those for uremic calciphylaxis, more efficient therapeutic measures and evidencebased recommendations are needed.

\section{KEYWORDS}

Calciphylaxis, leg ulcer, vascular calcification, sepsis

\section{INTRODUCTION}

Calciphylaxis is a rare disease characterized by arterial and arteriole calcification, soft tissue calcification, and thrombosis, resulting in ischaemia and skin necrosis ${ }^{[1]}$. It has a predilection for the lower extremities and areas rich in adipose tissue, although systemic involvement (internal organs or muscles) has also been described ${ }^{[1,2]}$. It is mainly diagnosed in patients with end-stage kidney disease (ESKD) on haemodialysis (HD). In this population, the reported incidence of calciphylaxis is $1 \%$, with a prevalence of $4 \%$ and 1 -year mortality of $45-$ $80 \%{ }^{[1-3]}$. Calciphylaxis in the absence of ESKD is called non-uremic calciphylaxis (NUC). In one systematic review on NUC which included 36 cases, the mortality rate was $52 \%$ and sepsis was responsible for $50 \%$ of deaths, most of them occurring 2 weeks to 1 year after diagnosis ${ }^{[2]}$. A common aetiology for these cases has not been described, although several risk factors and causative agents of NUC have been suggested (Table 1) ${ }^{[1,2,4,5]}$. This is probably related to its pathogenesis. Calciphylaxis is characterized by an insidious asymptomatic medial arteriolar calcification, resulting in clinical manifestations when vascular thrombosis occurs. Vascular calcification results from active cellular processes involved in biomineralization and the NFkB pathway, being regulated by several factors. Prothrombotic factors are additionally involved in the acute development of vascular occlusion ${ }^{[6]}$. 
The diagnosis of NUC requires a high level of suspicion and depends on the identification of the typical clinical features in the presence of risk factors and related conditions, while excluding clinical mimics (Table 2) ${ }^{[1,2,4,5]}$. It usually presents as a painful skin lesion with a livedoid pattern progressing to necrosis, frequently with a black stellate eschar and surrounding purpura that heals poorly and frequently becomes infected. A cutaneous biopsy is crucial to the diagnosis as findings that include tunica media calcification, fibrointimal hyperplasia of small dermal and subcutaneous arteries and arterioles with thrombosis, cutaneous ischaemia and panniculitis are pathognomonic ${ }^{[1-4]}$.

Therapeutic strategies for NUC mainly derive from those for uremic calciphylaxis. Possible triggers of occlusion and factors involved in the mineralization process should be identified and treated. Careful surgical or chemical debridement of the lesions, to remove eschar, bacterial biofilms and inflamed adipose tissue, is recommended ${ }^{[1-3]}$. Analgesia should be optimized ${ }^{[1,2]}$, and prompt treatment of sepsis is mandatory. Sodium thiosulfate (STS) has been gaining interest due to some successful outcomes, but most reports are on uremic calciphylaxis and this is as yet an off-label indication ${ }^{[1,2,7]}$. Antioxidation, vasodilation and chelation are the mechanisms thought to be responsible for its therapeutic action in calciphylaxis ${ }^{[8]}$. An ongoing clinical trial is evaluating the clinical efficacy of STS in uremic calciphylaxis ${ }^{[9]}$ and its results could support a new labelled indication. Other reported therapeutic alternatives are also associated with better outcomes, but most of the evidence is inferred from uremic calciphylaxis case reports and case series. These comprise hyperbaric oxygen therapy (HOT) [1,4,10], lanthanum carbonate ${ }^{[11]}$, statins ${ }^{[12]}$, bisphosphonates ${ }^{[13]}$, cinacalcet and sevelamer ${ }^{[14]}$. In selected cases, tissue plasminogen activator therapy to address the thrombotic component of the disease may be beneficial[ ${ }^{[15]}$. The most recommended strategy is to combine treatments to address both vascular calcification and thrombosis, and taking account of the metabolic and coagulation status of each patient.

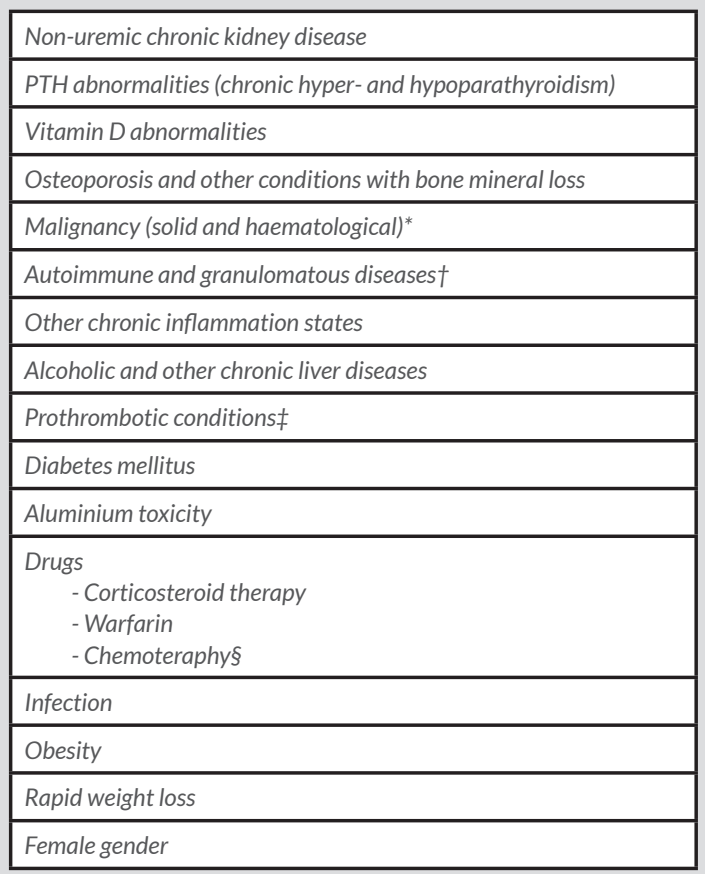

Table 1. Non-uremic calciphylaxis-associated factors and co-morbidities ${ }^{[1,2,4-6]}$ ${ }^{*}$ Cholangiocarcinoma, endometrial adenocarcinoma, malignant melanoma, metastatic breast cancer, metastatic parathyroid carcinoma with primary hyperparathyroidism, multiple myeloma and chronic myelocytic leukaemia. †Systemic lupus erythematosus, rheumatoid arthritis, giant cell arteritis, sarcoidosis and Crohn's disease. ¥Protein C and S deficiency, antithrombin III deficiency, cryofibrinogenemia and antiphospholipid antibody syndrome.

$\S C y c l o p h o s p h a m i d e$, adriamycin and fluorouracil.

\begin{tabular}{|l|}
\hline Atherosclerotic vascular disease \\
\hline Cholesterol embolization \\
\hline Nephrogenic systemic fibrosis \\
\hline Oxalate vasculopathy \\
\hline Purpura fulminans \\
\hline Vasculitis \\
\hline Warfarin necrosis \\
\hline
\end{tabular}




\section{CASE DESCRIPTION}

We present the case of a 78-year-old female patient with a known clinical history of obesity, arterial hypertension, diabetes mellitus (DM), dyslipidaemia, heart failure, atrial fibrillation, cardio-embolic stroke with left hemiparesis, chronic venous insufficiency and rheumatoid arthritis. Chronic medication included isophane insulin, metformin, digoxin, warfarin, ramipril, amlodipine, rosuvastatin, pantoprazole, prednisone and folic acid. Over a period of 8 weeks, the patient developed painful cutaneous ulcers initially on the right thigh, and later on both legs, characterized by irregular stellate borders, purpuric edges, and dry necrosis with a black eschar. The patient was hospitalized with a diagnosis of sepsis following disease progression and the development of infected ulcers.

Sepsis was treated with empiric antibiotic therapy as blood and wound cultures were sterile. Warfarin was switched to enoxaparin and prednisone reduced to $5 \mathrm{mg}$ daily. However, the skin ulcers remained resistant to wound care and new lesions appeared. Smaller violaceous non-ulcerated plaques and mottled to reticular purpura of surrounding skin were also present (Fig. 1). Therefore, a cutaneous punch biopsy on the edge of a skin ulcer was performed.

On histopathological examination, arteriole calcium deposition, thrombosis and panniculitis were observed (Fig. 2). These findings were diagnostic for calciphylaxis. The complementary investigation demonstrated a normal kidney function, normal levels of serum calcium, phosphorus, vitamin $\mathrm{D}$ and parathormone, an immune profile compatible with rheumatoid arthritis, a normal coagulation study, an arterial Doppler without flow disturbances, and a lower limb x-ray showing a calcified arterial net.

In light of the diagnosis of calciphylaxis, wound care was maintained with regular debridement, appropriate dressings and infection control, along with opioids for analgesia. A single session of HOT was performed, with poor patient tolerability. Treatment with STS was considered, but the patient died from septic shock 12 weeks after admission.
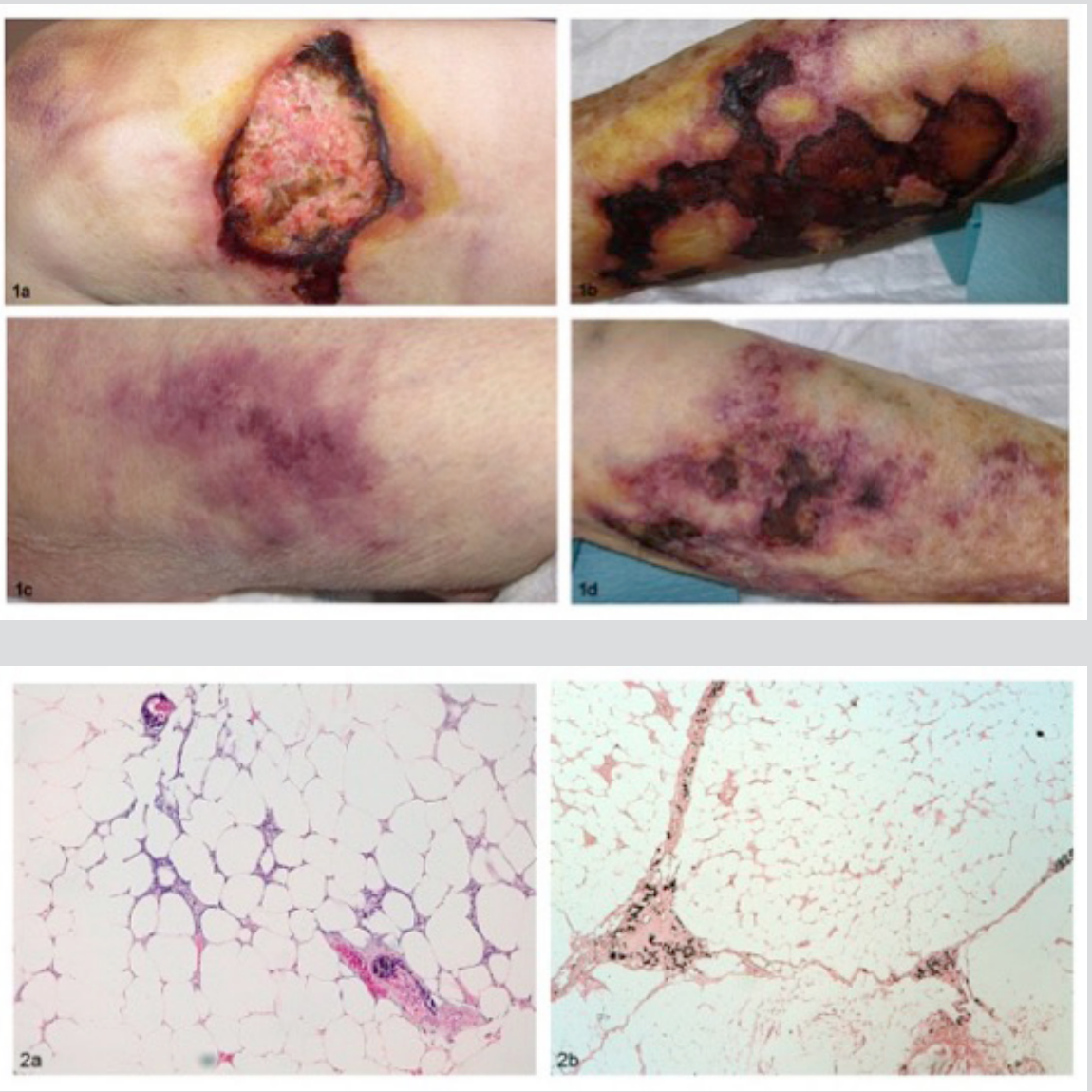

Figure 1. Painful stellate necrotic ulcers with mottled to reticular purpura of surrounding skin on both legs and the right thigh $(a, b, d)$; reticular violaceous stellate nonulcerated plaque on the left thigh (c)

Figure 2. Histology of cutaneous biopsy, with haematoxylineosin $(a, \times 100)$ and von Kossa $(b, \times 40)$ staining. There is calcification and occlusion of small arteries in subcutis, thickened adipose septa and extensive calcium depositionfindings consistent with the diagnosis of calciphylaxis 


\section{DISCUSSION}

NUC usually presents clinically with characteristic painful non-healing skin ulcers on the lower limbs, in the absence of ESKD, although there are some clinical differential diagnoses (Table 2). The definite diagnosis of calciphylaxis was established by the pathognomonic histopathological characteristics of a sample obtained through punch biopsy. The collaboration between multiple specialties, mainly Internal Medicine and Dermatology, a high level of suspicion and a good quality tissue sample for histopathological examination were fundamental for making the diagnosis in our patient.

In this case, female gender, obesity, DM, rheumatoid arthritis, and chronic warfarin and prednisone therapies may be have been possible triggers for NUC.

The management of this patient was insufficient to avoid death from sepsis, a common clinical course in reports of NUC. This disease represents a diagnostic and therapeutic challenge, with treatment currently limited to debridement, analgesia, and control of triggers and septic complications. Some therapeutic alternatives are mentioned in the literature, but mortality remains high and more efficient therapeutic measures and evidence-based recommendations are needed.

\section{REFERENCES}

1. Nigwekar SU, Kroshinsky D, Nazarian RM, Goverman J, Malhotra R, Jackson VA, et al. Calciphylaxis: risk factors, diagnosis, and treatment. Am J Kidney Dis 2015;66:133-146.

2. Nigwekar SU, Wolf M, Sterns RH, Hix JK. Calciphylaxis from nonuremic causes: a systematic review. Clin J Am Soc Nephrol 2008;3:1139-1143.

3. Wollina U. Update on cutaneous calciphylaxis. Indian J Dermatol 2013;58:87-92.

4. Lee JL, Naguwa SM, Cheema G, Gershwin ME. Recognizing calcific uremic arteriolopathy in autoimmune disease: an emerging mimicker of vasculitis. Autoimmun Rev 2008;7:638-643.

5. Marques SA, Kakuda AC, Mendaçolli TJ, Abbade LP, Marques ME. Calciphylaxis: a rare but potentially fatal event of chronic kidney disease. Case report. An Bras Dermatol 2013;88(6 Suppl 1):44-47.

6. Weenig RH. Pathogenesis of calciphylaxis: Hans Selye to nuclear factor kappa-B. J Am Acad Dermatol 2008;58:458-471.

7. Schlieper G, Brandenburg V, Ketteler M, Floege J. Sodium thiosulfate in the treatment of calcific uremic arteriolopathy. Nat Rev Nephrol 2009;5:539-543.

8. Vedvyas C, Winterfield LS, Vleugels RA. Calciphylaxis: a systematic review of existing and emerging therapies. J Am Acad Dermatol 2012;67:e253-260.

9. ISRCTN registry. ISRCTN73380053: A clinical trial with sodium thiosulfate for the treatment of calciphylaxis. Available from http://www.isrctn.com/ (accessed 13 Nov 2015).

10. Arenas MD, Gil MT, Gutiérrez MD, Malek T, Moledous A, Salinas A, Alvarez-Ude F. Management of calcific uremic arteriolopathy (calciphylaxis) with a combination of treatments, including hyperbaric oxygen therapy. Clin Nephrol 2008;70:261-264.

11. Chan MR, Ghandour F, Murali NS, Washburn M, Astor BC. Pilot study of the effect of lanthanum carbonate (Fosrenol $($ ) in patients with calciphylaxis: a Wisconsin Network for Health Research (WiNHR) study. J Nephrol Ther 2014;4:1000162.

12. Nigwekar SU, Bhan I, Turchin A, Skentzos SC, Hajhosseiny R, Steele D, Nazarian RM, et al. Statin use and calcific uremic arteriolopathy: a matched case-control study. Am J Nephrol 2013;37:325-332.

13. Ross EA. Evolution of treatment strategies for calciphylaxis. Am J Nephrol 2011;34:460-467.

14. Salmhofer H, Franzen M, Hitzl W, Koller J, Kreymann B, Fend F, et al. Multi-modal treatment of calciphylaxis with sodium-thiosulfate, cinacalcet and sevelamer including longterm data. Kidney Blood Press Res 2013;37:346-359.

15. el-Azhary RA, Arthur AK, Davis MD, McEvoy MT, Gibson LE, Weaver AL, et al. Retrospective analysis of tissue plasminogen activator as an adjuvant treatment for calciphylaxis. JAMA Dermatol 2013;149:63-67. 